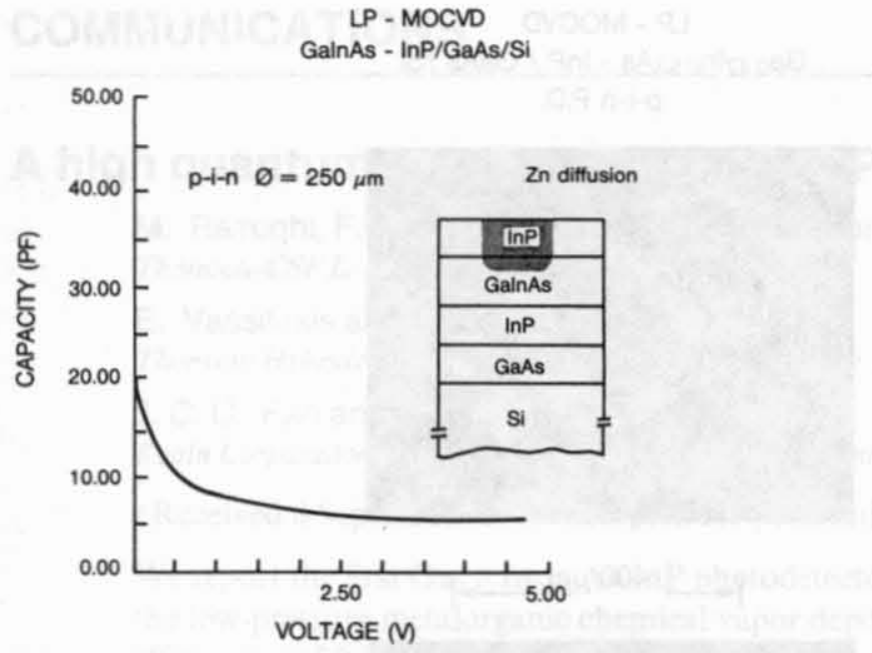

FIG. 5. The $C(V)$ characteristics of a GaInAs-InP $p-i-n$ photodiode on a $\mathrm{Si}$ substrate.

In summary, we report the first GaInAs-InP $p-i-n$ photodiode fabricated with material grown on a Si substrate using LP-MOCVD.

A reverse leakage current of $100 \mu \mathrm{A}$ at $-10-\mathrm{V}$ bias was measured for $250-\mu \mathrm{m}$-diam diodes. Quantum efficiencies of $0.34,0.88$, and $0.92 \mathrm{~A} / \mathrm{W}$ have been obtained at wavelengths of $0.8,1.3$, and $1.55 \mu \mathrm{m}$, respectively, for the devices without antireflection coating. A capacitance as low as $5 \mathrm{pF}$ for 250 $\mu \mathrm{m}$-diam diodes has been measured, providing evidence for the high-purity GaInAs active layer.

The authors would like to thank D. Le Guen, J. Antoine, D. S. Hill, and R. E. McCullough for technical assistance, and Dr. B. de Crémoux for reading the manuscript carefully.

\footnotetext{
'M. Razeghi, F. Omnes, M. Defour, and P. Maurel, Appl. Phys. Lett. 52, 209 (1988).

${ }^{2}$ M. Razeghi, R. Blondeau, M. Defour, F. Omnes, P. Maurel, and F. Brillouet, Appl. Phys. Lett. 53, 854 (1988).

${ }^{3}$ M. Razeghi, M. Defour, F. Omnes, P. Maurel, J. Chazelas, and F. Brillouet, Appl. Phys. Lett. 53, 725 (1988).

${ }^{4}$ M. Razeghi, M. Defour, R. Blondeau, F. Omnes, P. Maurel, O. Acher, F. Brillouet, J. C. C. Fan, and J. Salerno, Appl. Phys. Lett. 53, 2389 (1988). ${ }^{5}$ R. D. Hodron, R. R. Bradley, J. R. Riffat, T. B. Joyce, and R. H. Wallis, Electron. Lett. 23, 1094 (1987).

${ }^{6}$ J. P. Salerno, J. W. Lee, R. E. McCullough, and J. C. C. Fan, in Proceedings of MRS 1988 Spring Meeting, April 5-9, Reno, Nevada, edited by D. E. Clarke, C. W. Dropper, and C. T. Liu (1988), p. 10.

'P. Poulain, M. Razeghi, K. Kazmierski, R. Blondeau, and P. Philippe, Electron. Lett. 21, 442 (1985).
}

\title{
On the relaxation oscillation in copper-vapor lasers
}

\author{
Hiroshi Taniguchi and Hiroshi Saito \\ Department of Electronic Engineering, Faculty of Engineering, Iwate University, Ueda 4-3-5, \\ Morioka-shi 020, Japan
}

(Received 24 October 1988; accepted for publication 9 January 1989)

The possibility of relaxation oscillations in the laser output of a discharge-pumped copper

$(\mathrm{CuCl})$ vapor laser is explained qualitatively considering resonance-radiation trapping and

collisional effects for experimental results.

The phenomenon of relaxation oscillations has been observed in a variety of lasers, however, not so frequently for gas lasers. ${ }^{1,2}$ Relaxation oscillation is not generally observed because the decay rate of the upper laser level is large in most gas lasers compared to that in solid-state lasers, semiconductor lasers, and certain other laser systems in which the recovery time of the excited population inversion is substantially longer than the laser cavity decay time. ${ }^{1,3}$ On the other hand, in the gas-metal-vapor laser, the decay rate of the upper laser level varies over a wide range through the resonance-radiation trapping effect ${ }^{4}$ owing to the resonance transition between the ground and the upper laser level.

In this communication we describe qualitatively a possibility of relaxation oscillations in a pulsed discharge copper $(\mathrm{CuCl})$ vapor laser considering resonance-radiation trapping and collisional effects.
Miyazoe and Maeda ${ }^{5}$ report qualitative explanations for spiking phenomena in organic dye lasers with experimental results on rhodamine $6 \mathrm{G}$ dye laser output waveforms, which they analyze by means of a small-signal approximation (linear approximation) for the nonlinear coupled rate equations on the population inversion and the number of photons in the cavity. As a result of the analysis, they show that either spiking (oscillatory) or nonspiking (nonoscillatory) oscillation is observed, depending on the relative value between the fluorescence lifetime of the dye $\tau$ and the cavity decay rate $\gamma$ under a given excitation.

In copper-vapor lasers as mentioned above, the spontaneous decay rate (reciprocal lifetime) $\beta_{\text {res }}$ from the upper laser level to the ground level varies depending on the degree of resonance-radiation trapping, and the variation of $\beta_{\text {res }}$ will directly affect the spontaneous transition rate of the up- 

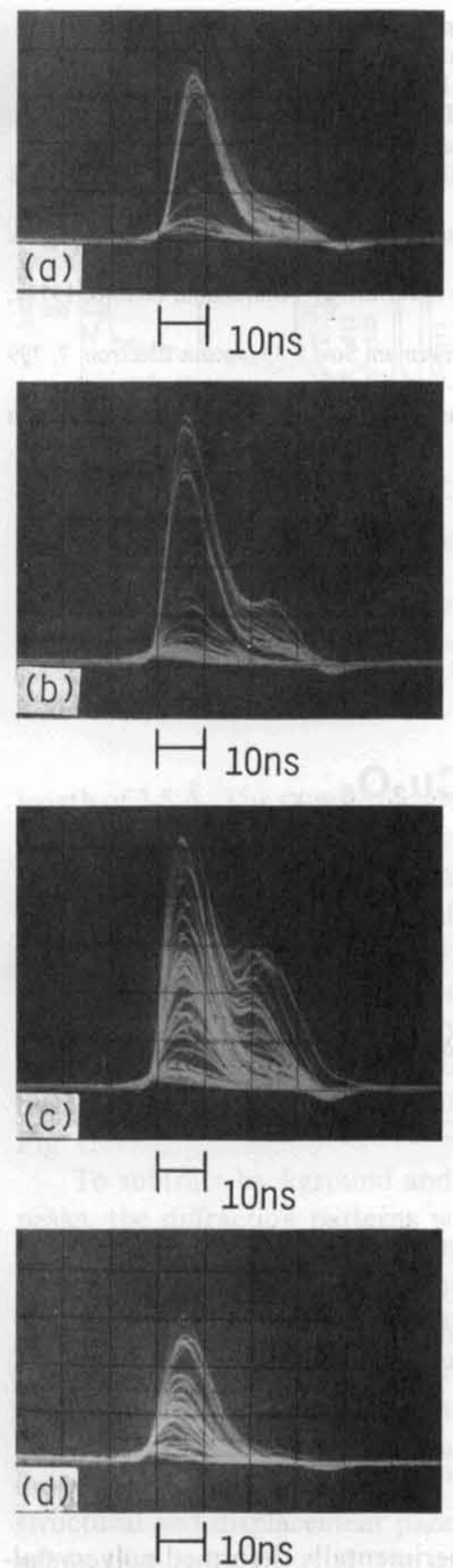

FIG. 1. Variation of the laser-output waveforms at $510.6 \mathrm{~nm}$ in the $\mathrm{CuCl}$ vapor laser with the gradual increase of the operating temperature. The operating temperature, $T$, is increased from (a) to (d) in this order. $T=340$, 350,360 , and $400^{\circ} \mathrm{C}$ in (a), (b), (c), and (d), respectively.

per laser level $\beta^{\prime}$. Assuming only two decay processes except the stimulated emission from the upper laser level to the ground level $\left(\beta_{\text {res }}\right)$ and to the lower laser level $\left(\beta_{\mathrm{ul}}\right)$, the transition rate $\beta^{\prime}$ is equal to the sum of $\beta_{\text {res }}$ and $\beta_{\mathrm{ul}}\left(\beta_{\mathrm{ul}}\right.$ is the spontaneous decay rate from the upper laser level to the lower laser level). $\beta^{\prime}$ is the reciprocal of the lifetime which corresponds to the fluorescence lifetime $\tau$ in the dye.

Considering a similar situation in the copper-vapor laser, a spiking oscillation seems to be probable just as in the dye laser. Both spiking and relaxation oscillation are similar effects which are exhibited by many (though not all) kinds of lasers. $^{6}$

Experiments were conducted with a copper-vapor laser using a $\mathrm{CuCl}$ lasant. The experimental setup was similar to that in previous reports. ${ }^{7,8}$ The laser tube was made of Tshaped Pyrex with a branch tube (hereafter described as reservoir) having $\mathrm{a} \sim 1.3-\mathrm{cm} \mathrm{i.} \mathrm{d.} \mathrm{and} \mathrm{a} \sim 20-\mathrm{cm}$ gain length. Small pieces of copper chloride $(\mathrm{CuCl})$ in the reservoir served as the copper-vapor source. $\mathrm{He}$ at a flow rate of 30 $\mathrm{cm}^{3} / \mathrm{min}$ was used as a buffer gas. Discharge products were purged from the tube by the vacuum pump. The internal pressure of the tube was kept at $\sim 4$ Torr. The optical cavity of $\sim 55-\mathrm{cm}$ spacing was formed by dielectrically coated flat mirrors, a high reflection mirror, and an output coupler with $43 \%$ transmittance $(\sim 57 \%$ reflectance $)$ at $510.6 \mathrm{~nm}$. The laser was driven in an aperiodic pulse train by successive pulse discharges from a capacitor at a voltage of about $5.4 \mathrm{kV}$ rms. ${ }^{7,8}$

If the cavity losses are determined only by the reflectance of the cavity mirror, under our experimental conditions, the cavity decay rate $\gamma$ is estimated to be about $10^{8} \mathrm{~s}^{-1}$. On the other hand, the decay rate of the upper laser level $\beta^{\prime}$ varies over a wide range from $1 / 9.6 \mathrm{~ns} \simeq 10^{8} \mathrm{~s}^{-1}$ without radiation trapping (i.e., the order of the decay rate from the upper laser level to the ground level in the isolated copper atom) to the order of $10^{6} \mathrm{~s}^{-1}$ with the complete radiation trapping (i.e., the order of the decay rate from the upper laser level to the lower laser level $\beta_{\mathrm{ul}}$ at the 510.6-nm line).

The higher the operating temperature, in a low copper concentration region, the more the decay rate $\beta^{\prime}$ decreases through resonance-radiation trapping. In a much higher copper concentration region the decay rate $\beta^{\prime}$ may increase again through presumable collisionlike processes which accompany the increase of the copper ground population (collisions increase the decay rate in proportion to ground populations $\left.{ }^{9}\right)$. Thus, the decay rate $\beta^{\prime}$ first decreases through resonance-radiation trapping and increases again through collisions as the operating temperature increases. This is considered to be supported by a rough estimation of $\beta^{\prime}$ variation for ground populations over the temperature range from 340 to $400^{\circ} \mathrm{C}$.

Measured waveforms of the laser output at $510.6 \mathrm{~nm}$ in the $\mathrm{CuCl}$ vapor laser are shown in Fig. 1. The operating temperature $T$ is increased in order from (a) to (d) (from $T=340$ to $400^{\circ} \mathrm{C} ; T$ crosses from $\beta^{\prime}$ decrease through resonance-radiation trapping to $\beta^{\prime}$ increase through collisions, in this region). The amplitude fluctuation in each figure is mainly due to the use of an ac driver with a free-running spark-gap switch of an air-blown type. ${ }^{7,8}$ A typical waveform of a discharge current was roughly triangular in shape with $\sim 100$-ns risetime, $\sim 150$-ns pulse duration, and 200300 -A peak in the first swing with the highest peak occurring after a few successive swings.

Increasing the operating temperature under a given excitation brings the laser to threshold and into the nonrelaxation oscillation region [ (a) near nonoscillatory]. Further temperature increase brings the laser to the relaxation oscillation region [ (b) and (c) oscillatory] with decreasing of $\beta^{\prime}$. Finally, the laser reaches the nonrelaxation oscillation 
region [ (d) nonoscillatory] again and falls below threshold as $\beta^{\prime}$ increases.

Qualitative considerations of the relaxation oscillation were presented as explainable treatments of the waveforms from a $\mathrm{CuCl}$-vapor laser. The considerations also explain qualitatively the laser waveforms observed in other works ${ }^{10,11}$ and in the other metal-vapor lasers. ${ }^{12}$

This work was partially supported by Grants-in-Aid for Scientific Research (Grant Nos. 61550269 and 63550281) from the Ministry of Education, Science, and Culture of Japan.

'L. W. Casperson and A. Yariv, IEEE J. Quantum Electron. QE-8, 69 (1972).
${ }^{2}$ K. Ueda and F. O. Shimizu, Jpn. J. Appl. Phys. 23, 1038 (1984).

${ }^{3}$ A. E. Siegman, Lasers (Oxford University, Oxford, 1986), p. 957.

${ }^{4}$ T. Holstein, Phys. Rev. 72, 1212 (1947); Phys. Rev. 73, 1159 (1951).

${ }^{5}$ Y. Miyazoe and M. Maeda, IEEE J. Quantum Electron. QE-7, 36 (1971).

${ }^{6}$ A. E. Siegman, Lasers (Oxford University, Oxford, 1986), p. 954.

${ }^{7}$ H. Saito and H. Taniguchi, IEEE J. Quantum Electron. QE-21, 1308 (1985).

${ }^{k}$ H. Saito, H. Taniguchi, H. Nozawa, and K. Owashi, Rev. Sci. Instrum. 56, 2248 (1985).

'A. Corney, Atomic and Laser Spectroscopy (Clarendon, Oxford, 1977), pp. $167-168$.

${ }^{10}$ A. A. Isaev and G. Yu. Lemmerman, Sov. J. Quantum Electron. 7, 799 (1977).

"M. A. Kazaryan, G. G. Petrash, and A. N. Trofimov, Sov. J. Quantum Electron. 10, 328 (1980).

${ }^{12}$ H. Taniguchi and H. Saito, J. Appl. Phys. 58, 4468 (1985).

\title{
Preferred orientation in experimentally deformed $\mathrm{YBa}_{2} \mathrm{Cu}_{3} \mathrm{O}_{6+x}$
}

\author{
Hans-Rudolf Wenk \\ Department of Geology and Geophysics, University of California, Berkeley, California 94720 \\ Jean Pannetier \\ Institut Laue-Langevin, 156X, 38042 Grenoble Cedex, France \\ Gilles Bussod. \\ Center for Materials Science, Los Alamos National Laboratory, Los Alamos, New Mexico 87545 \\ Alexander Pechenik \\ Department of Materials Science and Engineering, University of California, Los Angeles, California 90024
}

(Received 14 November 1988; accepted for publication 8 January 1989)

\begin{abstract}
Neutron diffraction data document that [001] axes of $\mathrm{YBa}_{2} \mathrm{Cu}_{3} \mathrm{O}_{6+x}$ in an experimentally deformed polycrystalline aggregate are preferentially aligned parallel to the compression direction. Preferred crystallographic orientation strongly affects oxygen stoichiometry results determined by structure refinement analysis of neutron diffraction data and appropriate corrections are necessary.
\end{abstract}

There have been several reports which suggest that pressed pellets of $\mathrm{YBa}_{2} \mathrm{Cu}_{3} \mathrm{O}_{6+x}$ polycrystals display grainshape anisotropy. ${ }^{1-4}$ In this communication we document strong crystallographic preferred orientation in a sample which has been deformed at high temperature and high confining pressure and which has anisotropic electrical properties. $^{4,5}$

A $\mathrm{YBa}_{2} \mathrm{Cu}_{3} \mathrm{O}_{6+x}$ powder was prepared using the amorphous citrate process. ${ }^{6}$ The material was then cold pressed to approximately $70 \mathrm{MPa}$ into cylinders of $6 \mathrm{~mm}$ diameter and $20 \mathrm{~mm}$ length using a steel dye, presintered in air at $800^{\circ} \mathrm{C}$ for $10 \mathrm{~h}$, then annealed at $450^{\circ} \mathrm{C}$ in oxygen for $20 \mathrm{~h}$. Following this procedure the $75 \%$ dense, $1-2-\mu \mathrm{m}$ grain-size powder compact was hydrostatically compacted under $1.0 \mathrm{GPa}$ confining pressure at $900^{\circ} \mathrm{C}$ for $1 \mathrm{~h}$. Subsequently it was deformed in a solid medium deformation apparatus at pressure and temperature and a strain rate of $10^{-5} \mathrm{~s}^{-1}$ and shortened $35 \%$ in axial compression. After deformation, the material is fully dense and textured. ${ }^{5}$
At this stage the experimentally deformed polycrystalline aggregate of $\mathrm{YBa}_{2} \mathrm{Cu}_{3} \mathrm{O}_{6+x}$ is oxygen deficient and nonsuperconducting. Following a 10 day anneal at $450^{\circ} \mathrm{C}$ in a pure $\mathrm{O}_{2}$ atmosphere it exhibits anisotropic properties. ${ }^{4,5}$ Most of the sample was used for resistivity and susceptibility measurements, and only a fragment of about $2 \times 3 \times 5 \mathrm{~mm}$ was available for the texture analysis. It is difficult to determine the preferred orientation of perovskite-related structures with $\mathrm{x}$-ray pole figure goniometry, because angular resolution is not adequate to resolve closely spaced diffraction peaks. Furthermore, transmission geometry, which could be used to measure the low angle (001) diffraction peak, is not very accurate due to strong absorption. We had the opportunity to use a position sensitive detector with the D1B instrument at the high flux reactor at the Institut Max von LauePaul Langevin (ILL), in Grenoble, France. This facility had previously been proved suitable for high-resolution texture analysis of complex geological materials. ${ }^{8-10}$ The radiation used is a monochromated beam of neutrons with a wave- 